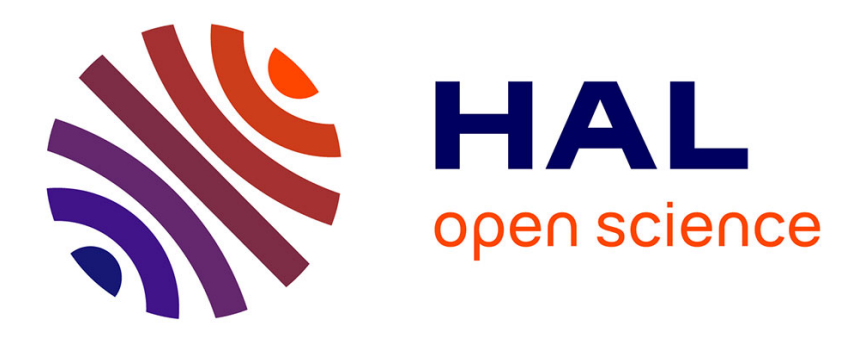

\title{
A new approach to the core and Weber set of multichoice games
}

Michel Grabisch, Lijue Xie

\section{To cite this version:}

Michel Grabisch, Lijue Xie. A new approach to the core and Weber set of multichoice games. Mathematical Methods of Operations Research, 2007, 66 (3), pp.491-512. 10.1007/s00186-007-0159-8 . halshs-00267933

\section{HAL Id: halshs-00267933 \\ https://shs.hal.science/halshs-00267933}

Submitted on 28 Mar 2008

HAL is a multi-disciplinary open access archive for the deposit and dissemination of scientific research documents, whether they are published or not. The documents may come from teaching and research institutions in France or abroad, or from public or private research centers.
L'archive ouverte pluridisciplinaire HAL, est destinée au dépôt et à la diffusion de documents scientifiques de niveau recherche, publiés ou non, émanant des établissements d'enseignement et de recherche français ou étrangers, des laboratoires publics ou privés. 


\title{
A new approach to the core and Weber set of multichoice games*
}

\author{
Michel GRABISCH†and Lijue XIE \\ Université Paris I - Panthéon-Sorbonne \\ email Michel.Grabisch@lip6.fr,Xielijue@yahoo.fr
}

February 13, 2007

\footnotetext{
${ }^{*}$ A preliminary and short version of this paper has been presented at 4 th Logic, Game Theory and Social Choice meeting, Caen, France, June 2005 [15].

${ }^{\dagger}$ Corresponding author. Mailing address: CERMSEM, Maison des Sciences Economiques, 106-112 Bd. de l'Hôpital, 75013 Paris, France. Tel (+33) 1-44-07-82-85, Fax (+33) 1-44-07-83-01
} 


\begin{abstract}
Multichoice games have been introduced by Hsiao and Raghavan as a generalization of classical cooperative games. An important notion in cooperative game theory is the core of the game, as it contains the rational imputations for players. We propose two definitions for the core of a multichoice game, the first one is called the precore and is a direct generalization of the classical definition. We show that the precore coincides with the definition proposed by Faigle, and that the set of imputations may be unbounded, which makes its application questionable. A second definition is proposed, imposing normalization at each level, causing the core to be a convex compact set. We study its properties, introducing balancedness and marginal worth vectors, and defining the Weber set and the pre-Weber set. We show that the classical properties of inclusion of the (pre)core into the (pre)-Weber set as well as their coincidence in the convex case remain valid. A last section makes a comparison with the core defined by van den Nouweland et al.
\end{abstract}

Keywords: multichoice game ; lattice ; core

\title{
1 Introduction
}

In cooperative game theory, one of the central problems is to select an efficient payoff vector, i.e., a way of sharing among players the total worth of a game, if they all join the grand coalition. There are two basic ways to do this. The first one is to define rational axioms such a sharing should satisfy, e.g., the null or dummy player axioms, symmetry, linearity, etc., with the hope that a unique sharing satisfies the set of axioms. This leads to several definitions of values or solution concepts, the most famous ones being the Shapley value [13] and the nucleolus [12]. The other way is to find a sharing so that no subcoalition has interest to form, that is, the sum of individual payoffs of all players for a given subcoalition is always greater or equal to the worth of this subcoalition. The set of such payoff vectors, whenever they exist, is called the core of the game. Classical results show under which conditions the core is nonempty, and give the structure of the core when the game is convex. A related notion is the Weber set (see, e.g., [3]), which is proven to always contain the core, with equality attained in case of convexity.

Many generalizations of the classical notion of cooperative game in characteristic form have appeared the last decade, in order to model in a more accurate way real situations. We may cite games with restricted cooperation of Faigle [5, 6], where admissible coalitions should satisfy some precedence constraints, multichoice games of Hsiao and Raghavan $[10,11]$, where each player is allowed to choose among a totally ordered set of actions, fuzzy games [2] which can be considered as a continuous extension of multichoice games, bi-cooperative games of Bilbao [1], where the worth of a coalition depends on whether some remaining agents form an opponent coalition, etc. All these examples can be thought as particular instances of games defined on a distributive lattice structure, see a general exposition of this in [8,9], and also Faigle and Kern [6].

A natural question is then to try to define the core of a game on a lattice, or of some of the most useful examples cited above. In this paper, we focus on multichoice games (Section 3), and propose a definition of the core and the Weber set. As it will be shown in Section 4, the situation appears to be more complex than for the classical 
case, although similar results still hold. A first immediate generalization of the classical definition leads to what we call the precore, which happens to be a convex polyhedron with infinite directions. We propose to call core a particular convex compact subset of it, satisfying some normalization constraint. Similarly to the classical case, we call preWeber set the convex hull of the additive games induced by marginal worth vectors, and the Weber set is a particular subset of it. We show that in case of convexity, we still have equality between the core and the Weber set, and between the convex compact part of the precore and the pre-Weber set. Moreover, the inclusion of the core into the Weber set holds in any case, as well as the inclusion of the convex compact part of the precore into the pre-Weber set.

We compare our results with previous works of Faigle and van den Nouweland et al. in Section 5. We find that our precore is the core defined by Faigle [5]. The relation with the core of van den Nouweland [14] appears to be less simple, since it is a subset of our precore.

The interest of the definition of the core we propose is that it keeps the original meaning of the set of payoff vectors such that no subcoalition can achieve more by itself, subcoalition being here replaced by the more general notion of participation profile with unequal levels of participation. Also, our solution possesses better and more natural mathematical properties since they are very close to those of the classical case.

We begin by setting our notations and recall classical results in Section 2. Then Section 3 presents the basic material for multichoice games. In Section 4, we give our definitions of the core and the Weber set, and study their properties. Lastly, Section 5 compares our approach with previous works.

\section{Background}

Throughout this paper, we consider a finite set of players $N:=\{1, \ldots, n\}$. A (classical) TU game on $N$ is a function $v: 2^{N} \rightarrow \mathbb{R}$ such that $v(\emptyset)=0$. The set of classical TU games on $N$ is denoted by $\mathcal{G}\left(2^{N}\right)$.

A game is said to be convex if $v(S \cup T)+v(S \cap T) \geq v(S)+v(T)$, for all $S, T \subseteq N$. A game is said to be additive if $v(S \cup T)=v(S)+v(T)$, for all disjoint $S, T \subseteq N$. If $v$ is additive, it suffices to know only $v(\{i\}), i \in N$. A game is said to be monotone if $v(S) \leq v(T)$, for all $S, T \subseteq N$ such that $S \subseteq T$.

An $n$-dimensional real-valued vector $\phi \in \mathbb{R}^{n}$ is called a payoff vector of a given game $v$. In addition, if $\phi(N):=\sum_{i \in N} \phi_{i}=v(N)$, we call it an efficient payoff vector, or pre-imputation. Note that an additive game uniquely defines a pre-imputation, and viceversa, hence the notation $\phi(N)$, or more generally $\phi(S):=\sum_{i \in S} \phi_{i}, S \subseteq N$.

It is useful for the sequel to reconsider the above definitions in terms of lattices. We begin by a short introduction on lattices. A a partially ordered set $(P, \leq)$ or a poset for short, is a set $P$ endowed with a binary relation $\leq$ satisfying reflexivity, antisymmetry and transitivity. For any two element $x, y \in P$, we call $x \vee y$ the supremum of $x$ and $y$ if it is the least element of all those greater than $x$ and $y$, and $x \wedge y$ the infimum of $x$ and $y$ if it is the greatest element of all those less than $x$ and $y$. The top element $\top$ of $(P, \leq)$ is the greatest element of $(P, \leq)$, and the bottom element $\perp$ of $(P, \leq)$ is the least element of $(P, \leq)$. For any $x, y \in P, x \vee y, x \wedge y, \top$ and $\perp$ are unique whenever they exist. If 
for any $x, y \in P$, both $x \vee y$ and $x \wedge y$ exist, the poset $(P, \leq)$ is called a lattice. For any $x, y \in(P, \leq)$ such that $x \leq y$, we define $[x, y]:=\{z \in P \mid x \leq z \leq y\}$.

A classical TU game is a real-valued function defined on the lattice $\left(2^{N}, \subseteq\right.$ ) (called a Boolean lattice), vanishing at the bottom $\perp=\emptyset$ of the lattice. Union and intersection are the sup $\vee$ and inf $\wedge$ operations of the lattice, so that a general form of convexity writes $v(x \vee y)+v(x \wedge y) \geq v(x)+v(y)$. This is usually called supermodularity.

For any lattice $(L, \leq)$, a chain is a totally ordered sequence of elements of $L$, e.g., $x<y<z<\cdots$. A chain is maximal if no superset of it is again a chain. In a finite distributive lattice, maximal chains going from bottom $\perp$ to top $\top$ have all the same length, and are of the form $\perp \prec x \prec y \prec z \prec \cdots \prec \top$, where $x \prec y$ means that $x<y$ and there is no $z \in L$ such that $x<z<y$ ( $x$ is covered by $y$ ).

In the Boolean lattice $\left(2^{N}, \subseteq\right)$, a maximal chain between $\emptyset$ and $N$ is of the form $\emptyset \subset\{i\} \subset\{i, j\} \subset \cdots \subset N$, and hence is associated in a bijective way with a permutation $\pi$ on $N$, defining the order in which elements $i, j, \ldots$ appear. We adopt the following notation: for any permutation $\pi$ on $N$, we define the maximal chain

$$
A_{0}^{\pi}:=\emptyset \subset A_{1}^{\pi}:=\{\pi(1)\} \subset A_{2}^{\pi}:=\{\pi(1), \pi(2)\} \subset \cdots \subset A_{n}^{\pi}:=N
$$

with $A_{i}^{\pi}:=\{\pi(1), \ldots, \pi(i)\}$. The marginal worth vector $x^{\pi}(v)$ is defined by:

$$
x_{\pi(i)}^{\pi}(v):=v\left(A_{i}^{\pi}\right)-v\left(A_{i-1}^{\pi}\right), \quad i=1, \ldots, n .
$$

The set of marginal worth vectors is denoted $\mathcal{M}(v)$. The $i$ th coordinate represents the marginal contribution of player $i$ in the chain.

Let us remark that any marginal worth vector $x^{\pi}$ is an efficient payoff vector, and thus defines an additive game $\phi^{\pi}$ by

$$
\phi^{\pi}(\{i\})=x_{i}^{\pi}, \quad \forall i \in N,
$$

with the property that $\phi^{\pi}\left(A_{i}^{\pi}\right)=v\left(A_{i}^{\pi}\right), i=0, \ldots, n$.

The Weber set of $v$ is the convex hull of the set of marginal worth vectors

$$
\mathcal{W}(v):=\operatorname{co}(\mathcal{M}(v))
$$

For any convex set $C$, we denote by $\operatorname{Ext}(C)$ the set of its extreme points (vertices).

A collection $\mathcal{B}$ of nonempty subsets of $N$ is balanced if there exist positive coefficients $\mu(S), S \in \mathcal{B}$, such that

$$
\sum_{S \ni i} \mu(S)=1, \quad \forall i \in N
$$

Any partition $\left\{P_{1}, \ldots, P_{k}\right\}$ of $N$ is a balanced collection, with coefficients $\mu\left(P_{i}\right)=1, \forall i$. A game $v$ is balanced if for every balanced collection $\mathcal{B}$ with coefficients $\mu(S), S \in \mathcal{B}$, it holds

$$
\sum_{S \in \mathcal{B}} \mu(S) v(S) \leq v(N)
$$

The core of $v$ is a set of efficient payoff vectors such that no subcoalition can achieve more by itself:

$$
\mathcal{C}(v):=\left\{\phi \in \mathbb{R}^{n} \mid \phi(N)=v(N) \text { and } \phi(A) \geq v(A), \forall A \subseteq N\right\}
$$


with $\phi(A):=\sum_{i \in A} \phi_{i}$. Equivalently, it is the set of additive games greater than $v$ and coinciding on $N$. Whenever nonempty, the core is a convex set. It is reduced to the singleton $\{v\}$ if the game is additive.

The following proposition summarizes well-known results.

Proposition 1 Let $v$ be a game on $N$. The following holds.

- (i) $\mathcal{C}(v) \subseteq \mathcal{W}(v)$.

- (ii) $\mathcal{C}(v) \neq \emptyset$ if and only if $v$ is balanced.

- (iii) $v$ is convex if and only if $\operatorname{Ext}(\mathcal{C}(v))=\mathcal{M}(v)$, or equivalently $\mathcal{C}(v)=\mathcal{W}(v)$.

\section{Games on lattices and multichoice games}

We give a brief introduction to games defined on lattices (see [8, 9] for a more detailed presentation). We consider again a set of players $N:=\{1, \ldots, n\}$. Let $L_{1}, \ldots, L_{n}$ be finite distributive lattices representing the partially ordered set of actions each player can perform, and consider their product $L:=L_{1} \times \cdots \times L_{n}$, together with the product order, i.e., for $x:=\left(x_{1}, \ldots, x_{n}\right), y:=\left(y_{1}, \ldots, y_{n}\right) \in L, x \leq y$ iff $x_{i} \leq y_{i}$ for $i=1, \ldots, n$. Supremum and infimum are also defined coordinatewise. The top and bottom of $L$ are denoted $T, \perp$.

A game on $L$ is any function $v: L \rightarrow \mathbb{R}$ such that $v(\perp)=0$. We denote by $\mathcal{G}(L)$ the set of games on $L$. Specifically, $L_{i}$ is the ordered set of possible actions player $i$ has at his disposal. The bottom element of $L_{i}$ indicates no participation to the game by player $i$, while the top element indicates full participation. A given $x \in L$ is then a profile of participation of all players, indicating for each player the action chosen. This replaces the notion of coalition.

We give some examples, to recover known concepts. Classical cooperative games correspond to $L_{i}=\{0,1\}$ for all $i \in N$, "0" indicating no participation and "1" full participation; hence $L$ is the Boolean lattice $2^{N}$. Multichoice games of Hsiao and Raghavan correspond to $L_{i}=\{0,1, \ldots, l\}$ for all $i \in N$, where $1,2, \ldots, l$ are the ordered participation levels of a player. Bi-cooperative games may correspond to the case $L_{i}=\{0,1,2\}$, $i \in N$, where "0" corresponds to participation against, "2" to participation in favor, and "1" no participation, although a better notation would be $L_{i}=\{-1,0,1\}$. In fact, bi-cooperative games, as well as ternary voting games of Felsenthal and Machover [7], correspond to the simplest case of bipolar games, as defined by Grabisch in [8], and are not isomorphic to multichoice games with $l=2$ (this point is however out of the scope of this paper).

In this paper, we focus on multichoice games; however our definition is slightly more general than the original one of Hsiao and Raghavan.

Definition 1 Let $L_{i}:=\left\{0,1,2, \ldots, l_{i}\right\}$ endowed with the natural order, $i \in N$, and consider the product lattice $L=L_{1} \times \cdots \times L_{n} . A$ multichoice game is any function $v: L \rightarrow \mathbb{R}$ such that $v(0,0, \ldots, 0)=0$. A l-choice game is a multichoice game where $l_{1}=l_{2}=\cdots=l_{n}=: l$. 
Note that $(0,0, \ldots, 0)$ is the bottom element $\perp$ of $L$, and " 0 " denotes non participation. The top element of $L$ is denoted $T:=\left(l_{1}, \ldots, l_{n}\right)$ as usual. Any $x:=\left(x_{1}, \ldots, x_{n}\right) \in L$ is a participation profile, indicating the participation level of each player. We recall that (see above):

$$
\begin{aligned}
x \leq y & \Leftrightarrow x_{1} \leq y_{1} \text { and } x_{2} \leq y_{2} \text { and } \cdots \text { and } x_{n} \leq y_{n} \\
x \vee y & :=\left(x_{1} \vee y_{1}, \ldots, x_{n} \vee y_{n}\right) \\
x \wedge y & :=\left(x_{1} \wedge y_{1}, \ldots, x_{n} \wedge y_{n}\right)
\end{aligned}
$$

We will use sometimes the notation $\left(x_{-i}, k_{i}\right)$ to denote the participation profile where player $i$ plays at level $k \in L_{i}$, and other players play at levels defined by the participation profile $x \in L$. We put for convenience $x_{-i} \in L_{-i}:=\prod_{j \neq i} L_{j}$. A particular case is the situation where the $i$-th player plays at the $k$-th action level, $k>0$, the others doing nothing, which is denoted by $\left(0_{-i}, k_{i}\right)$.

In multichoice games, a payoff vector is defined for any player participating at any level. Following van den Nouweland et al. [14], we introduce $M:=\left\{(i, k) \mid i \in N, k \in L_{i}\right\}$, so that a payoff vector $\phi$ is an element of $\mathbb{R}^{M}$, whose coordinates are denoted by $\phi_{(i, k)}$, with the convention $\phi_{(i, 0)}=0, \forall i \in N$. Similarly to the classical case, we put for any $x \in L$

$$
\phi(x):=\sum_{i \in N} \phi_{\left(i, x_{i}\right)} .
$$

For convenience, we also introduce $M^{*}:=\left\{(i, k) \mid i \in N, k \in L_{i} \backslash\{0\}\right\}$. Evidently, any vector in $\mathbb{R}^{M^{*}}$ defines a payoff vector, and vice-versa. Simlarly, a payoff vector could be considered as an alement of $\mathbb{R}^{\sum_{j \in N} l_{j}}$.

We denote by $\mathcal{C}_{m}(L)$ the set of maximal chains of the lattice $L$. Its cardinality is known to be (see, e.g., Faigle and Kern [6])

$$
\left|\mathcal{C}_{m}(L)\right|=\frac{\left(l_{1}+l_{2}+\cdots+l_{n}\right) !}{l_{1} ! l_{2} ! \cdots l_{n} !} .
$$

Next definitions follow those for games on (general) lattices.

Definition 2 Let $v$ be a multichoice game on L. Then

(i) $v$ is monotone if $x \leq y$ implies $v(x) \leq v(y)$.

(ii) $v$ is convex if $v(x \vee y)+v(x \wedge y) \geq v(x)+v(y)$, for all $x, y \in L$.

(iii) $v$ is additive if for every $x, y \in L$ such that $x \wedge y=\perp$, it holds $v(x \vee y)=v(x)+v(y)$.

Proposition 2 A game $v$ is additive if and only if for all $x \in L, v(x)=\sum_{i=1}^{n} v_{i}\left(x_{i}\right)$ holds, with $v_{i}: L_{i} \longrightarrow \mathbb{R}$, and $v_{i}(k):=v\left(0_{-i}, k_{i}\right)$, for all $i \in N$ and $k \in L_{i}$.

Proof: Assuming additivity of $v$, we have $v\left(0_{-\{i, j\}}, k_{i}, k_{j}^{\prime}\right)=v_{i}(k)+v_{j}\left(k^{\prime}\right)$. Hence, by repeating this process, we finally get for any $x \in L, v(x)=\sum_{i=1}^{n} v_{i}\left(x_{i}\right)$.

Reciprocally, if $v$ can be written under the above form, then it is additive since taking $x, y \in L$ such that $x \wedge y=\perp$ implies that for each $i \in N$, either $x_{i}=0_{i}$ or $y_{i}=0_{i}$. Hence $v(x \vee y)=v(x)+v(y)$ holds. 
REMARK 1:

1. Proposition 2 shows the equivalence, as in the classical case, between payoff vectors and additive games. It justifies the notation $\phi(x)$ introduced in (1).

2. The concepts introduced in Def. 2 remain applicable for games on any lattice $L=\prod_{i=1}^{n} L_{i}$. However, Proposition 2 does not hold in general. Hence, the correspondence between payoff vectors and additive games may be lost.

\section{The core and Weber set of multichoice games}

The following definition is a direct transposition of the classical definition of the core for TU games.

Definition 3 The precore of a multichoice game $v$ on $L$ is defined by

$$
\mathcal{P C}(v):=\left\{\phi \in \mathbb{R}^{M} \mid \phi(x) \geq v(x), \forall x \in L, \text { and } \phi(\top)=v(\top)\right\}
$$

Note that any $\phi \in \mathcal{P C}(v)$ is an efficient payoff vector (or additive game by Remark 1). Clearly, the precore is a convex set. Let us consider the following example as an illustration.

ExAmPLE 1: We consider a 2-choice game with two players, hence $L:=$ $\{0,1,2\}^{2}$. The conditions on $\phi$ to be element of the precore write:

$$
\begin{aligned}
(2,2) & =\phi_{(1,2)}+\phi_{(2,2)} & =v(2,2) \\
\phi(2,0) & =\phi_{(1,2)} & \geq v(2,0) \\
\phi(0,2) & =\phi_{(2,2)} & \geq v(0,2) \\
\phi(1,1) & =\phi_{(1,1)}+\phi_{(2,1)} & \geq v(1,1) \\
\phi(1,0) & =\phi_{(1,1)} & \geq v(1,0) \\
\phi(0,1) & =\phi_{(2,1)} & \geq v(0,1) .
\end{aligned}
$$

Remark that $\phi(1,0)$ and $\phi(0,1)$ may be taken arbitrarily large. This shows that in general, $\mathcal{P C}(v)$ is an unbounded convex polyhedron, hence it can be written as the Minkovski sum of a polytope $\mathcal{P C}^{F}(v):=\operatorname{co}(\operatorname{Ext}(\mathcal{P C}(v)))$ and a cone (see, e.g., Ziegler [16]).

This is not acceptable since individual payoff vectors of some players may become infinite. This drawback could be avoided if normalization occurs not only on the last level, i.e., $\phi(T)=v(T)$, but also on each level $1,2, \ldots, l$. This motivates the next definition.

Definition 4 The core of a multichoice game $v$ on $L$ is defined as:

$$
\begin{aligned}
\mathcal{C}(v):=\left\{\phi \in \mathbb{R}^{M} \mid\right. & \phi(x) \geq v(x), \forall x \in L, \\
& \text { and } \left.\phi\left(k \wedge l_{1}, \ldots, k \wedge l_{n}\right)=v\left(k \wedge l_{1}, \ldots, k \wedge l_{n}\right), k=1, \ldots, \max _{j} l_{j}\right\} .
\end{aligned}
$$


Note that the precore always contains the core. For $l$-choice games, the normalization condition simplifies into $\phi(k, k, \ldots, k)=\nu(k, k, \ldots, k), k=1, \ldots, l$. Obviously the core is a convex set, where all variables are bounded, hence it is a convex polytope.

We give an interpretation of the definition. A given $x \in L$ represents the profile of participation of all players, supposed to achieve a common task, so that $x$ could be thought of as a team of players with different participation levels. A basic requirement for the task to be achieved properly is that all players of the team should have equal level of participation (this is a kind of fairness condition: no one works less than the others), and ideally, this level of participation should be as high as possible, despite the fact that players may have the tendency to play at the lowest possible level (the less work, the better). If the core of $v$ is nonempty, it is possible to achieve this goal by rewarding players with a payoff $\phi \in \mathcal{C}(v)$.

Let us explain this with the simple case of a 2-choice game $v$ on $N$. If everybody plays at level 2 , the total amount to share is $v(2,2, \ldots, 2)$, and any player $i \in N$ will receive $\phi_{(i, 2)}$. There is no interest to have a profile of participation $x$ mixing levels 2,1 and 0 (including at least one player at level 2), since $\phi(x) \geq v(x)$ for all such profiles.

Suppose now that nobody wants to play at level 2 , but at most at level 1 . Then $\phi$ favors the formation of the profile $(1,1, \ldots, 1)$, and in this case the total value to be shared is $v(1,1, \ldots, 1)$, hence the normalization condition.

Lastly, suppose that $v$ is monotonic. Then $v(2,2, \ldots, 2) \geq v(1,1, \ldots, 1)$, so that the total amount shared by players will be higher if they play at level 2 rather than at level 1. Hence, in case of monotonicity, our definition of the core is an incentive to play at the highest level.

We introduce now balancedness for the core and precore. A collection $\mathcal{B}$ of elements of $L \backslash\{\perp\}$ is pre-balanced if there exist positive coefficients $\mu(x), x \in \mathcal{B}$, such that

$$
\begin{aligned}
& \sum_{x \in \mathcal{B} \mid x_{i}=l_{i}} \mu(x)=1, \text { for all } i \in N, \\
& \sum_{x \in \mathcal{B} \mid x_{i}=k} \mu(x)=0, \text { for all } k=1, \ldots, l_{i}-1, \quad i \in N
\end{aligned}
$$

ExAmple 2: Let $n=4$ and $l_{1}=l_{2}=l_{3}=l_{4}=3$. The following is a pre-balanced collection

$$
\mathcal{B}=\{(3,3,3,0),(0,3,3,3),(3,0,0,3)\}
$$

with coefficients $\mu(3,3,3,0)=\mu(0,3,3,3)=\mu(3,0,0,3)=0.5$.

Definition 5 A multi-choice game $v$ is pre-balanced if for every pre-balanced collection $\mathcal{B}$ of elements of $L \backslash\{\perp\}$ with coefficients $\mu(x), x \in \mathcal{B}$, it holds

$$
\sum_{x \in \mathcal{B}} \mu(x) v(x) \leq v(\top)
$$

Proposition 3 A multi-choice game has a nonempty precore if and only if it is prebalanced. 
Proof: Nonemptiness of the precore is equivalent to find a payoff vector $\phi \in \mathbb{R}^{M^{*}}$ such that

$$
\sum_{i \in N, x_{i}>0} \phi_{\left(i, x_{i}\right)} \geq v(x), \forall x \in L, \text { and } \phi(\top)=\sum_{i \in N} \phi_{\left(i, l_{i}\right)}=v(\top),
$$

which in turn is equivalent to the fact that the following linear program

$$
\min z=\sum_{i \in N} \phi_{\left(i, l_{i}\right)} \text { subject to } \sum_{i \in N, x_{i}>0} \phi_{\left(i, x_{i}\right)} \geq v(x), \forall x \in L
$$

has an optimal solution with value $z^{*} \leq v(T)$. Indeed, any optimal solution is such that $\sum_{i \in N} \phi_{\left(i, l_{i}\right)}=v(\top)$, and so lies in the precore. Conversely, any $\phi$ in the precore satisfies the contraints, and is such that $\phi(\top)=v(\top)$. Hence $z^{*} \leq v(\top)$ is true.

Its dual problem is

$$
\begin{aligned}
& \max _{\mu} q=\sum_{x \in L} \mu(x) v(x) \text { subject to } \\
& \mu(x) \geq 0, \quad x \in L \\
& \sum_{x_{-i} \in L_{-i}} \mu\left(x_{-i}, l_{i}\right)=1, \quad i \in N \\
& \sum_{x_{-i} \in L_{-i}} \mu\left(x_{-i}, k_{i}\right)=0, \quad 1 \leq k \leq l_{i}-1, \quad i \in N .
\end{aligned}
$$

By the duality theorem, $z^{*}=q^{*} \leq v(\top)$. Hence, nonemptiness of the precore is equivalent to the existence of an optimal solution $\mu^{*}$ such that $\sum_{x \in L} \mu^{*}(x) v(x) \leq v(\top)$ and satisfying the constraints, which means exactly that $v$ is pre-balanced.

A collection $\mathcal{B}$ of elements of $L \backslash\{\perp\}$ is balanced if there exist positive coefficients $\mu(x), x \in \mathcal{B}$, such that

$$
\begin{aligned}
& \sum_{x \in \mathcal{B} \mid x_{i}=l_{i}} \mu(x)=\left(\max _{j} l_{j}\right)-l_{i}+1, \text { for all } i \in N \\
& \sum_{x \in \mathcal{B} \mid x_{i}=k} \mu(x)=1, \text { for all } k=1, \ldots, l_{i}-1, \quad i \in N .
\end{aligned}
$$

Remark that for $l$-choice games, the above two conditions simplify into $\sum_{x \in \mathcal{B} \mid x_{i}=k} \mu(x)=$ 1 , for all $k=1, \ldots, l$ and $i \in N$.

EXAMPLE 3: Let us take as before $n=4$ and $l_{1}=l_{2}=l_{3}=l_{4}=3$. The following is a balanced collection

$$
\begin{aligned}
\mathcal{B}=\{(2,0,3,1),(1,2,0,0),(0,1,2,2),(2,3,3,1) \\
(2,0,3,1),(3,0,1,3),(0,1,0,2),(0.3,2,0)\}
\end{aligned}
$$

with $\mu(2,0,3,1)=0.5, \mu(1,2,0,0)=\mu(3,0,1,3)=1, \mu(0,1,2,2)=\mu(2,3,3,1)=$ $\mu(2,0,3,1)=0.25, \mu(0,1,0,2)=\mu(0.3,2,0)=0.75$. 
Definition 6 A multichoice game $v$ is balanced if for every balanced collection $\mathcal{B}$ of elements of $L \backslash\{\perp\}$ with coefficients $\mu(x), x \in \mathcal{B}$, it holds

$$
\sum_{x \in \mathcal{B}} \mu(x) v(x) \leq \sum_{k=1}^{\max _{j} l_{j}} v\left(k \wedge l_{1}, \ldots, k \wedge l_{n}\right)
$$

Proposition 4 A multichoice game has a nonempty core if and only if it is balanced.

Proof: The proof is similar to the one of Prop. 3. Nonemptiness of the core is equivalent to finding a payoff vector $\phi \in \mathbb{R}^{M^{*}}$ such that

$$
\begin{aligned}
& \sum_{i \in N, x_{i}>0} \phi_{\left(i, x_{i}\right)} \geq v(x), \forall x \in L, \text { and } \phi\left(k \wedge l_{1}, \ldots, k \wedge l_{n}\right)= \\
& \qquad \sum_{i \in N} \phi_{\left(i, k \wedge l_{i}\right)}=v\left(k \wedge l_{1}, \ldots, k \wedge l_{n}\right), \forall k=1, \ldots, \max _{j} l_{j},
\end{aligned}
$$

which is equivalent to the fact that the following linear program:

$$
\begin{aligned}
\min z=\sum_{i \in N}\left[\sum_{1 \leq k<l_{i}} \phi_{\left(i, k_{i}\right)}+\left(\left(\max _{j} l_{j}\right)-l_{i}+1\right) \phi_{\left(i, l_{i}\right)}\right] \\
\text { subject to } \sum_{i \in N, x_{i}>0} \phi_{\left(i, x_{i}\right)} \geq v(x), \forall x \in L,
\end{aligned}
$$

has an optimal solution $z^{*} \leq \sum_{k=1}^{\max _{j} l_{j}} v\left(k \wedge l_{1}, \ldots, k \wedge l_{n}\right)$. Its dual problem is

$$
\begin{aligned}
& \max _{\mu} q=\sum_{x \in L} \mu(x) v(x) \text { subject to } \\
& \mu(x) \geq 0, \quad \forall x \in L \\
& \sum_{x_{-i} \in L_{-i}} \mu\left(x_{-i}, l_{i}\right)=\left(\max _{j} l_{j}\right)-l_{i}+1, \quad i \in N \\
& \sum_{x_{-i} \in L_{-i}} \mu\left(x_{-i}, k_{i}\right)=1, \quad k=1, \ldots, l_{i}-1, \forall i \in N .
\end{aligned}
$$

The existence of an optimal solution is equivalent to the fact that $v$ is balanced.

Consider a maximal chain $C:=\left\{x^{0}:=\perp<x^{1}<\cdots<x^{\sum_{j=1}^{n} l_{j}}:=\top\right\}$ in $L$. Let us remark that between $x^{i-1}$ and $x^{i}$, only one coordinate has changed, and has been increased by exactly 1 . Hence, to each maximal chain one can associate the mapping $\sigma:\left\{1, \ldots, \sum_{j} l_{j}\right\} \rightarrow M^{*}$, defined by $\sigma(i)=(j, k), k \in L_{j} \backslash\{0\}$, which means that at the $i$ th element of the chain, the level of player $j$ has been raised from $k-1$ to $k$, in symbols:

$$
x^{i}=x^{i-1} \vee\left(0_{-j}, k_{j}\right) .
$$

Assimilating $(j, k)$ to $\left(0_{-j}, k_{j}\right)$, one can write $x^{i}=\bigvee_{p=1}^{i} \sigma(p)$, for any element of the chain. Note that all mappings $\sigma$ (which are in fact permutations on $\left\{1, \ldots, \sum_{j} l_{j}\right\}$ ) may 
not correspond to a maximal chain. For this, $\sigma$ must fulfill: $\left(j, k^{\prime}\right)>(j, k)$ implies $i^{\prime}>i$, where $\sigma(i):=(j, k)$ and $\sigma\left(i^{\prime}\right):=\left(j, k^{\prime}\right)$ (multipermutation).

We define the marginal worth vector $\psi^{C} \in \mathbb{R}^{M^{*}}$ associated to $C$ and $v$ as:

$$
\psi_{(j, k)}^{C}:=v\left(x^{\sigma^{-1}(j, k)}\right)-v\left(x^{\sigma^{-1}(j, k)-1}\right), \quad \forall(j, k) \in M^{*} .
$$

From this, we define the payoff vector $\phi^{C} \in \mathbb{R}^{M}$ by:

$$
\phi_{(j, k)}^{C}:=\sum_{p=1}^{k} \psi_{(j, p)}^{C}, \quad j \in N, k \in L_{j} \backslash\{0\},
$$

and $\phi_{(j, 0)}^{C}=0, \forall j \in N$. The set of all such payoff vectors $\phi^{C}$ for all maximal chains is denoted by $\mathcal{P} \mathcal{M}(v)$.

A fundamental property of $\phi^{C}$ is that it coincides with $v$ on $C$.

Lemma 1 Let $C$ be a maximal chain on L. For every $x \in C, \phi^{C}(x)=v(x)$.

Proof: Consider a maximal chain $C:=\left\{x^{0}:=\perp<x^{1}<\cdots<x^{\sum_{j=1}^{n} l_{j}}:=\top\right\}$ in $L$, and its associated mapping $\sigma$. Assume $x=\left(k_{1}, \ldots, k_{n}\right), \sigma(i):=(j, k)$. We have:

$$
\phi_{(j, k)}^{C}=\sum_{p=1}^{k} \psi_{(j, p)}^{C}=\sum_{p=1}^{k}\left[v\left(x^{\sigma^{-1}(j, p)}\right)-v\left(x^{\sigma^{-1}(j, p)-1}\right)\right],
$$

and

$$
\begin{aligned}
\phi^{C}(x)=\sum_{j=1}^{n} \phi_{(j, k)}^{C} & =\sum_{j=1}^{n} \sum_{p=1}^{k} \psi_{(j, p)}^{C}=\sum_{j=1}^{n}\left[\sum_{p=1}^{k}\left[v\left(x^{\sigma^{-1}(j, p)}\right)-v\left(x^{\sigma^{-1}(j, p)-1}\right)\right]\right] \\
& =v\left(x^{\sigma^{-1}(j, k)}\right)=v(x) .
\end{aligned}
$$

Definition 7 The pre-Weber set $\mathcal{P W}(v)$ of $v$ is defined as the convex hull of all payoff vectors in $\mathcal{P} \mathcal{M}(v)$ :

$$
\mathcal{P W}(v):=\operatorname{co}(\mathcal{P} \mathcal{M}(v)) .
$$

Theorem 1 If a multichoice game $v$ is convex, then any payoff vector in $\mathcal{P} \mathcal{M}(v)$ is a vertex of the precore:

$$
\mathcal{P} \mathcal{M}(v) \subseteq \operatorname{Ext}(\mathcal{P C})(v) .
$$

Proof: Let $C:=\left\{x^{0}:=\perp<x^{1}<\cdots<x^{\sum_{j=1}^{n} l_{j}}:=\top\right\}$ be a maximal chain in $L$, and consider $\phi^{C}$.

We first show that $\phi^{C} \in \mathcal{P C}(v)$. We take any $x \in L$ and show that $\phi^{C}(x) \geq v(x)$. By Lemma 1 , the property is true for any $x \in C$, so let us suppose $x \notin C$. Then there exists a unique $i$ such that $x^{i+1}>x$ and $x^{i} \ngtr x$. Moreover, there exists a unique $j \in N$ such that $x_{j}^{i+1}=x_{j}$ and $x_{j}^{i}=x_{j}-1$, where $x_{j}$ is the $j$ th coordinate of $x$. In summary, we have the following situation: 


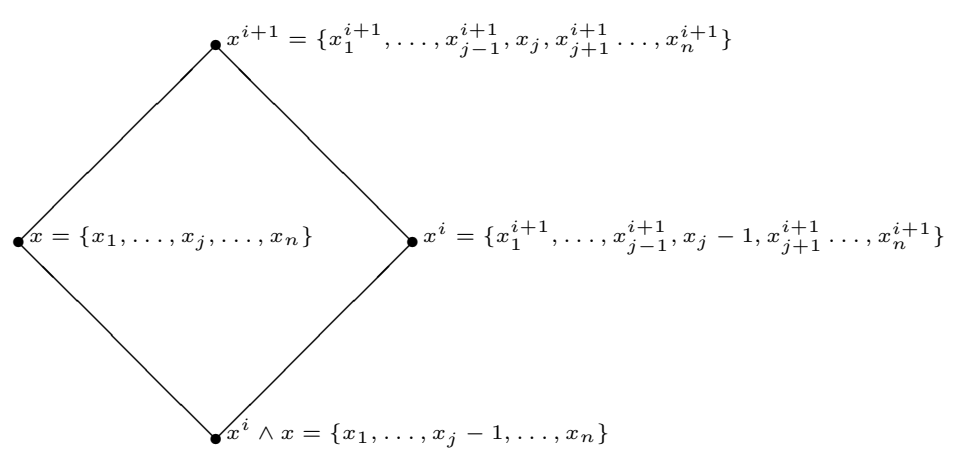

By convexity of $v$, we have

$$
v\left(x^{i+1}\right)-v\left(x^{i}\right) \geq v(x)-v\left(x^{i} \wedge x\right) .
$$

Note that if $x^{i} \wedge x \in C$, we are done since $\phi^{C} \equiv v$ on $C$, so that the above inequality writes $\phi^{C}\left(x^{i+1}\right)-\phi^{C}\left(x^{i}\right) \geq v(x)-\phi^{C}\left(x^{i} \wedge x\right)$. By definition of $\phi^{C}$, we have $\phi^{C}(x)=$ $\phi^{C}\left(x^{i+1}\right)-\phi^{C}\left(x^{i}\right)+\phi^{C}\left(x^{i} \wedge x\right)$, which leads to $\phi^{C}(x) \geq v(x)$.

If $x^{i} \wedge x \notin C$, we construct a decreasing sequence $x^{\prime}, x^{\prime \prime}, \ldots$ which will meet the chain $C$. Specifically, let us consider $x^{\prime}:=x^{i} \wedge x$, and $i^{\prime}$ such that $x^{i^{\prime}+1}>x^{\prime}$ and $x^{i^{\prime}} \ngtr x^{\prime}$. The same inequality as (3) holds with $x^{\prime}, i^{\prime}$ replacing $x, i$. If $x^{i^{\prime}} \wedge x^{\prime} \in C$, the result can be proven as above. Indeed, we get:

$$
\begin{aligned}
v\left(x^{i+1}\right)-v\left(x^{i}\right) & \geq v(x)-v\left(x^{i} \wedge x\right) \\
v\left(x^{i^{\prime}+1}\right)-v\left(x^{i^{\prime}}\right) & \geq v\left(x^{i} \wedge x\right)-v\left(x^{i^{i}} \wedge x^{i} \wedge x\right) .
\end{aligned}
$$

Summing the inequalities leads to

$$
v\left(x^{i+1}\right)-v\left(x^{i}\right)+v\left(x^{i^{\prime}+1}\right)-v\left(x^{i^{\prime}}\right) \geq v(x)-v\left(x^{i^{\prime}} \wedge x^{i} \wedge x\right)
$$

which is equivalent to

$$
\phi^{C}\left(x^{i+1}\right)-\phi^{C}\left(x^{i}\right)+\phi^{C}\left(x^{i^{\prime}+1}\right)-\phi^{C}\left(x^{i^{\prime}}\right) \geq v(x)-\phi^{C}\left(x^{i^{\prime}} \wedge x^{i} \wedge x\right) .
$$

By definition of $\phi^{C}$

$$
\begin{aligned}
\phi^{C}\left(x^{\prime}\right) & =\phi^{C}\left(x^{i^{\prime}+1}\right)+\phi^{C}\left(x^{i^{\prime}} \wedge x^{i} \wedge x\right)-\phi^{C}\left(x^{i^{\prime}}\right) \\
\phi^{C}(x) & =\phi^{C}\left(x^{i+1}\right)+\phi^{C}\left(x^{\prime}\right)-\phi^{C}\left(x^{i}\right)
\end{aligned}
$$

so that

$$
\phi^{C}(x)=\phi^{C}\left(x^{i+1}\right)+\phi^{C}\left(x^{i^{\prime}+1}\right)+\phi^{C}\left(x^{i^{\prime}} \wedge x^{i} \wedge x\right)-\phi^{C}\left(x^{i^{\prime}}\right)-\phi^{C}\left(x^{i}\right) .
$$

Replacing into (4) leads to $\phi^{C}(x) \geq v(x)$.

If $x^{i^{\prime}} \wedge x^{\prime} \notin C$, we consider $x^{\prime \prime}:=x^{i^{\prime}} \wedge x^{\prime}$ and $i^{\prime \prime}$ accordingly. Clearly the sequence $x, x^{\prime}, x^{\prime \prime}, \ldots$ is strictly decreasing and will meet the chain $C$ due to finiteness of $L$, latest at bottom of $L$ since $\perp \in C$. Repeating the above process proves that $\phi^{C}(x) \geq v(x)$, $\forall x \in L$.

It remains to show that $\phi^{C}$ is a vertex of the precore. Suppose there exist payoff vectors $\phi_{1}, \phi_{2} \neq \phi^{C} \in \mathcal{P C}(v)$, and $\lambda \in(0,1)$ such that $\phi^{C}=\lambda \phi_{1}+(1-\lambda) \phi_{2}$. Because we have $\phi^{C}\left(x^{i}\right)=v\left(x^{i}\right)$ for any $x^{i} \in C$, we have $v\left(x^{i}\right)=\lambda \phi_{1}\left(x^{i}\right)+(1-\lambda) \phi_{2}\left(x^{i}\right)$. But $\phi_{k}\left(x^{i}\right) \geq v\left(x^{i}\right)$ for all $x^{i} \in C, k=1,2$, hence necessarily $\phi_{1}\left(x^{i}\right)=\phi_{2}\left(x^{i}\right)=v\left(x^{i}\right)$, i.e., $\phi_{1}=\phi_{2}=\phi^{C}$, a contradiction. 
Definition $8 A$ restricted maximal chain $C_{r}=\left\{x^{0}:=\perp<x^{1}<\cdots<x^{\sum_{i=1}^{n} l_{i}}:=\top\right\}$ in $L$ is a maximal chain passing by all $\left(k \wedge l_{1}, \ldots, k \wedge l_{n}\right), k=1, \ldots, \max _{j} l_{j}$. The set of all restricted maximal chains is denoted by $\mathcal{C}_{m}^{r}(L)$.

Proposition 5 Suppose $l_{1} \leq l_{2} \cdots \leq l_{n}$. The number of restricted maximal chains is given by:

$$
\begin{aligned}
\left|\mathcal{C}_{m}^{r}(L)\right| & =(n !)^{l_{1}} \times((n-1) !)^{l_{2}-l_{1}} \times \cdots \times(1 !)^{l_{n}-l_{n-1}} \\
& =n^{l_{1}}(n-1)^{l_{2}} \cdots 1^{l_{n}} .
\end{aligned}
$$

If $l_{1}=l_{2}=\cdots=l_{n}=: l$ (l-choice game), then $\left|\mathcal{C}_{m}^{r}(L)\right|=(n !)^{l}$.

Proof: Let us first prove the statement for $l$-choice games. Any restricted maximal chain has the form $\left\{(0, \ldots, 0), x^{1}, \ldots,(1, \ldots, 1), x^{n+1}, \ldots,(2, \ldots, 2), x^{2 n+1}, \ldots,(l, \ldots, l)\right\}$. Between $(k, \ldots, k)$ and $(k+1, \ldots, k+1)$, for $k=0, \ldots, l-1$, there are $n$ ! maximal chains since the sublattice $[(k, \ldots, k),(k+1, \ldots, k+1)]$ is the Boolean lattice $2^{N}$. Hence, $\left|\mathcal{C}_{m}^{r}(L)\right|=(n !)^{l}$.

We turn to the general case. Any restricted maximal chain has the form $\left\{\perp, x^{1}, \ldots,\left(l_{1}, \ldots, l_{1}\right), x^{l_{1} n+1}, \ldots,\left(l_{1}, l_{2}, \ldots, l_{2}\right), x^{l_{1}+l_{2}(n-1)+1}, \ldots,\left(l_{1}, l_{2}, l_{3}, \ldots, l_{3}\right), \ldots\right.$, $\left.\left(l_{1}, \ldots, l_{n}\right)\right\}$. In $L$, the sublattice $\left[\perp,\left(l_{1}, \ldots, l_{1}\right)\right]:=\left\{x \in L \mid \perp \leq x \leq\left(l_{1}, \ldots, l_{n}\right)\right\}$ corresponds to a $l_{1}$-choice game with $n$ players, hence the number of restricted maximal chains is $(n !)^{l_{1}}$. The sublattice $\left[\left(l_{1}, \ldots, l_{1}\right),\left(l_{1}, l_{2}, \ldots, l_{2}\right)\right]$ corresponds to a $\left(l_{2}-l_{1}\right)$-choice game with $n-1$ players, hence it has $((n-1) !)^{l_{2}-l_{1}}$ restricted maximal chains. Continuing the process till the sublattice $\left[\left(l_{1}, \ldots, l_{n-1}, l_{n-1}\right),\left(l_{1}, \ldots, l_{n}\right)\right]$ proves the formula.

The marginal worth vector $\psi^{C_{r}}$ associated to a restricted chain $C_{r}$ is defined as before, as well as the associated payoff vector $\phi^{C_{r}}$. Note that $\phi^{C_{r}} \equiv v$ on $C_{r}$ still holds. The set of payoff vectors $\phi^{C_{r}}$ for all restricted maximal chains is denoted $\mathcal{M}(v)$.

Restricted marginal worth vectors will play a central role in the sequel. Note that for any participation profile of a restricted maximal chain, the difference of levels of participation among players does not exceed 1 . In other words, if player $i$ at level $k-1$ want to reach level $k$, he must wait till all other players have reached level $k-1$.

Definition 9 The Weber set is defined as the convex hull of all payoff vectors $\phi^{C_{r}}$ :

$$
\mathcal{W}(v):=\operatorname{co}(\mathcal{M}(v))
$$

Theorem 2 If a multichoice game $v$ is convex, then any payoff vector in $\mathcal{M}(v)$ is a vertex of the core:

$$
\mathcal{M}(v) \subseteq \operatorname{Ext}(\mathcal{C})(v)
$$

Proof: Consider a restricted maximal chain $C_{r}$ and its associated payoff vector $\phi^{C_{r}}$. We know by Th. 1 that it is a vertex of the precore, and since $\phi^{C_{r}}$ coincides with $v$ on $C_{r}$, it has the property $\phi^{C_{r}}\left(k \wedge l_{1}, \ldots, k \wedge l_{n}\right)=v\left(k \wedge l_{1}, \ldots, k \wedge l_{n}\right), k=1, \ldots, \max _{j} l_{j}$, hence it belongs to the core and is a vertex of it.

As a corollary of Th. 1 and 2, we obtain: 
Corollary 1 Let $v$ be a convex multichoice game. Then

1. $\mathcal{P} \mathcal{W}(v) \subseteq \mathcal{P C}^{F}(v)$

2. $\mathcal{W}(v) \subseteq \mathcal{C}(v)$

Our aim is now to study the inclusion of the core in the Weber set, like in the classical case.

Theorem 3 For any multichoice game $v$, the polytope of the precore is included in the pre-Weber set, i.e, $\mathcal{P C}^{F}(v) \subseteq \mathcal{P W}(v)$.

The proof relies on the following lemmas.

Lemma 2 Let $\phi, y$ be two vectors in $\mathbb{R}^{\sum_{j} l_{j}}$, and $\phi$ be considered as a payoff vector. Then their inner product is:

$$
\phi \cdot y=\sum_{i=1}^{\sum_{j} l_{j}} \phi\left(x^{i}\right)\left(Y_{\sigma(i)}-Y_{\sigma(i+1)}\right)
$$

for any maximal chain $\left\{x^{0}:=\perp, x^{1}, x^{2}, \ldots, \top\right\}$ and corresponding mapping $\sigma$, and $Y_{(j, k)}:=$ $\sum_{p=k}^{l_{j}} y_{(j, p)}$, for all $(j, k) \in M^{*}$, and $Y_{\sigma\left(\sum_{j} l_{j}+1\right)}:=0$.

Proof: We have, letting $\sigma(i):=(j(i), k(i))$ :

$$
\begin{aligned}
\sum_{i=1}^{\sum_{j} l_{j}} \phi\left(x^{i}\right)\left(Y_{\sigma(i)}-Y_{\sigma(i+1)}\right) & =\sum_{i=1}^{\sum_{j} l_{j}} Y_{\sigma(i)}\left(\phi\left(x^{i}\right)-\phi\left(x^{i-1}\right)\right) \\
& =\sum_{i=1}^{\sum_{j} l_{j}}\left(\sum_{p=k(i)}^{l_{j(i)}} y_{(j(i), p)}\right)\left(\phi_{(j(i), k(i))}-\phi_{(j(i), k(i)-1)}\right)(\phi \text { is a payoff vector }) \\
& =\sum_{i=1}^{\sum_{j} l_{j}} \phi_{(j(i), k(i))}\left(\sum_{p=k(i)}^{l_{j(i)}} y_{(j(i), p)}-\sum_{p=k(i)+1}^{l_{j(i)}} y_{(j(i), p)}\right) \\
= & \sum_{i=1}^{\sum_{j} l_{j}} \phi_{(j(i), k(i))} y_{(j(i), k(i))} \\
= & \sum_{i=1}^{\sum_{j} l_{j}} \phi_{\sigma(i)} y_{\sigma(i)}=\phi \cdot y .
\end{aligned}
$$

Lemma 3 Given any $y \in \mathbb{R}^{\sum_{j} l_{j}}$, there exists a maximal chain with corresponding mapping $\sigma$ such that $Y_{\sigma(1)} \geq \cdots \geq Y_{\sigma\left(\sum_{j} l_{j}\right)}$. 
Proof: We partition $\left\{1, \ldots, \sum_{j} l_{j}\right\}$ into $n$ disjoint intervals $I_{i}:=\left[\sum_{j=1}^{i} l_{j}+1, \sum_{j=1}^{i+1} l_{j}\right]$, $i=0, \ldots, n-1$, corresponding to the levels in $L_{1}, \ldots, L_{n}$. In each interval $I_{i}$, compute

$$
Y_{k}:=\sum_{p=k}^{\sum_{j=1}^{i+1} l_{j}} y_{p}, \quad k \in I_{i}
$$

Take a permutation $\pi$ such that $Y_{\pi(1)} \geq \cdots \geq Y_{\pi\left(\sum_{j} l_{j}\right)}$. Then if $\pi(k) \in I_{i}$, put $\sigma(k):=$ $(i+1, p)$, where $p$ is the first level in $L_{i+1}$ not already chosen, and put $Y_{\sigma(k)}:=Y_{\pi(k)}$. Then by construction $\sigma$ is compatible with a chain, and $Y_{\sigma(i)}$ is non increasing with $i$.

EXAmple 4: We illustrate Lemma 3 . Let $n=2$ and $l_{1}=2, l_{2}=3$, and consider $y=(0.1,0.7,0.5,0.3,0.2)$. The partition of $\{1,2,3,4,5\}$ gives $\{\{1,2\},\{3,4,5\}\}$. Hence the computation of $Y$ gives $Y=(0.8,0.7,1,0.5,0.2)$, and we get:

$$
\sigma(1)=1_{2}, \quad \sigma(2)=1_{1}, \quad \sigma(3)=2_{1}, \quad \sigma(4)=2_{2}, \quad \sigma(5)=3_{2},
$$

which defines the maximal chain $\{(0,1),(1,1),(2,1),(2,2),(2,3)\}$. We have effectively $Y_{\sigma(1)}=Y_{1_{2}}=0.5+0.3+0.2=1, Y_{\sigma(2)}=Y_{1_{1}}=0.1+0.7=0.8$, etc.

Let us now prove Th. 3. Our proof uses the separation theorem for closed convex sets, similarly to the proof of Derks for proving the inclusion of the (classical) core into the Weber set [4].

Proof: Suppose there exists a payoff vector $\phi$ in $\mathcal{P C}^{F}(v)$, but not belonging to the pre-Weber set. By the separation theorem, it should exist $y \in \mathbb{R}^{\sum_{j} l_{j}}$ such that for any payoff vector $\phi^{\prime}$ in the pre-Weber set, $\phi \cdot y<\phi^{\prime} \cdot y$.

Let us consider such a $y$ and $\phi$. Then, for any maximal chain and corresponding mapping $\sigma$, using Lemma 2, we get:

$$
\phi \cdot y=\sum_{i=1}^{\sum_{j} l_{j}} \phi\left(x^{i}\right)\left(Y_{\sigma(i)}-Y_{\sigma(i+1)}\right) .
$$

Let us choose a maximal chain $C$ with mapping $\sigma$ such that $Y_{\sigma(i)}-Y_{\sigma(i+1)} \geq 0, i=$ $1, \ldots, \sum_{j} l_{j}$. This is always possible by Lemma 3. Considering that $\phi$ is in the precore, and that the payoff vector $\phi^{C}$ in the pre-Weber set satisfies $\phi^{C} \equiv v$ on $C$, we deduce:

$$
\begin{aligned}
\phi \cdot y & \geq \sum_{i=1}^{\sum_{j} l_{j}} v\left(x^{i}\right)\left(Y_{\sigma(i)}-Y_{\sigma(i+1)}\right) \\
& =\sum_{i=1}^{\sum_{j} l_{j}} \phi^{C}\left(x^{i}\right)\left(Y_{\sigma(i)}-Y_{\sigma(i+1)}\right)
\end{aligned}
$$

a contradiction. Hence, $\mathcal{P C}^{F}(v)$ is included in $\mathcal{P} \mathcal{W}(v)$. 
Theorem 4 For any multichoice game $v$, the core is included in the Weber set, i.e., $\mathcal{C}(v) \subseteq \mathcal{W}(v)$.

We need the following lemma.

Lemma 4 Given any $y \in \mathbb{R}^{\sum_{j} l_{j}}$, there exists a restricted maximal chain $\left\{\perp, x^{1}, x^{2}, \ldots, \top\right\}$ with corresponding mapping $\sigma_{r}$ such that $Y_{\sigma_{r}(i)}-Y_{\sigma_{r}(i+1)} \geq 0$, for each $i \in\left\{1, \ldots, \sum_{j} l_{j}\right\}$ such that $x^{i}$ and $x^{i+1}$ belong to the sublattice $\left[\left((k-1) \wedge l_{1}, \ldots,(k-1) \wedge l_{n}\right),\left(k \wedge l_{1}, \ldots, k \wedge l_{n}\right)\right]$, $x^{i} \neq\left((k-1) \wedge l_{1}, \ldots,(k-1) \wedge l_{n}\right)$ for some $k \in\left\{1, \ldots, \max _{j} l_{j}\right\}$.

Proof: Given $y$, by Lemma 3, there exists a maximal chain $C$ in $L$ with mapping $\sigma$ such that $Y$ is non increasing along it. It is easy to build a restricted maximal chain $C_{r}$ from $C$ so that the above condition is satisfied: starting from $\perp$, put $\sigma_{r}(1):=1_{j}=\sigma(1)$, then $\sigma_{r}(2)=1_{j^{\prime}}$ if $\sigma^{-1}\left(1_{j^{\prime}}\right)<\sigma^{-1}\left(1_{k}\right)$ for all $k \neq j, j^{\prime}$, and so on till $C_{r}$ is defined in the first sublattice $[\perp,(1,1, \ldots, 1)]$. Then from $(1, \ldots, 1)$ proceed similarly, i.e., $\sigma_{r}(n+1)=2_{j}$ if $\sigma^{-1}\left(2_{j}\right)<\sigma^{-1}\left(2_{k}\right)$ for all $k \neq j$, etc.

EXAmple 5: Let $n=2, l_{1}=3, l_{2}=4$, and consider $y$ defined below.

\begin{tabular}{|c||c|c|c|c|c|c|c|}
\hline$(i, k)$ & $(1,1)$ & $(1,2)$ & $(1,3)$ & $(2,1)$ & $(2,2)$ & $(2,3)$ & $(2,4)$ \\
\hline$y_{(i, k)}$ & 0.1 & 0.6 & 0.1 & 0.3 & 0.5 & 0.7 & 0.2 \\
$Y_{(i, k)}$ & 0.8 & 0.7 & 0.1 & 1.7 & 1.4 & 0.9 & 0.2 \\
\hline
\end{tabular}

Then the corresponding maximal chain is defined by:

$$
\begin{gathered}
\sigma(1)=(2,1), \quad \sigma(2)=(2,2), \quad \sigma(3)=(2,3), \quad \sigma(4)=(1,1), \quad \sigma(5)=(1,2), \\
\sigma(6)=(2,4), \quad \sigma(7)=(1,3) .
\end{gathered}
$$

Applying Lemma 4, we find the following maximal restricted chain:

$$
\begin{gathered}
\sigma_{r}(1)=(2,1), \quad \sigma_{r}(2)=(1,1), \quad \sigma_{r}(3)=(2,2), \quad \sigma_{r}(4)=(1,2), \quad \sigma_{r}(5)=(2,3), \\
\sigma_{r}(6)=(1,3), \quad \sigma_{r}(7)=(2,4) .
\end{gathered}
$$

One can verify that

$$
\begin{aligned}
& Y_{(2,1)}=1.7 \geq Y_{(1,1)}=0.8, \\
& Y_{(2,2)}=1.4 \geq Y_{(1,2)}=0.7, \\
& Y_{(2,3)}=0.9 \geq Y_{(1,3)}=0.1, \\
& Y_{(2,4)}=0.2,
\end{aligned}
$$

as expected.

Proof: (of Th. 4) We use the same technique as for Th. 3. Assume $\phi \in \mathcal{C}(v) \backslash$ $\mathcal{W}(v)$. Then it should exist $y \in \mathbb{R}^{\sum_{j} l_{j}}$ such that for any element $\phi^{\prime}$ in the Weber set, $\phi \cdot y<\phi^{\prime} \cdot y$. Considering such a $y$, we know by Lemma 4 that there exists a restricted maximal chain $C_{r}$ with corresponding mapping $\sigma_{r}$ such that $Y$ is non increasing on each 
sublattice $\left[\left((k-1) \wedge l_{1}, \ldots,(k-1) \wedge l_{n}\right),\left(k \wedge l_{1}, \ldots, k \wedge l_{n}\right)\right]$, except its bottom element $\left((k-1) \wedge l_{1}, \ldots,(k-1) \wedge l_{n}\right)$. Let us decompose $\phi \cdot y$ as follows:

$$
\phi \cdot y=\sum_{i=1}^{\sum_{j} l_{j}} \phi_{i} y_{i}=\sum_{k=1}^{\max _{j} l_{j}} \sum_{i=1}^{n(k)} \phi_{\sum_{j=1}^{k-1} n(j)+i} y_{\sum_{j=1}^{k-1} n(j)+i}
$$

where $n(k)=\left|\left\{j \mid k \wedge l_{j}=k\right\}\right|$, i.e., we have decomposed the sum according to the different levels. In each term of the right hand, we apply Lemma 2. This gives:

$$
\begin{aligned}
\phi \cdot y=\sum_{k=1}^{\max _{j} l_{j}}\left[\sum_{i=1}^{n(k)-1} \phi\left(x^{\sum_{j=1}^{k-1} n(j)+i}\right)\left(Y_{\sigma_{r}\left(\sum_{j=1}^{k-1} n(j)+i\right)}-Y_{\sigma_{r}\left(\sum_{j=1}^{k-1} n(j)+i+1\right)}\right)\right. \\
\left.+\phi\left(x^{\sum_{j=1}^{k} n(j)}\right)\left(Y_{\sigma_{r}\left(\sum_{j=1}^{k} n(j)\right)}-Y_{\sigma_{r}\left(\sum_{j=1}^{k} n(j)+1\right)}\right)\right] .
\end{aligned}
$$

Observe that $x^{\sum_{j=1}^{k} n(j)}=\left(k \wedge l_{1}, \ldots, k \wedge l_{n}\right)$ by definition of $C_{r}$. Since $\phi \in \mathcal{C}(v)$ implies that $\phi\left(k \wedge l_{1}, \ldots, k \wedge l_{n}\right)=v\left(k \wedge l_{1}, \ldots, k \wedge l_{n}\right)$, and by Lemma 4 it follows that

$$
\begin{aligned}
& \phi \cdot y \geq \quad \sum_{k=1}^{\max _{j} l_{j}}\left[\sum_{i=1}^{n(k)-1} v\left(x^{\sum_{j=1}^{k-1} n(j)+i}\right)\left(Y_{\sigma_{r}\left(\sum_{j=1}^{k-1} n(j)+i\right)}-Y_{\sigma_{r}\left(\sum_{j=1}^{k-1} n(j)+i+1\right)}\right)\right. \\
& \left.+v\left(x^{\sum_{j=1}^{k} n(j)}\right)\left(Y_{\sigma_{r}\left(\sum_{j=1}^{k} n(j)\right)}-Y_{\sigma_{r}\left(\sum_{j=1}^{k} n(j)+1\right)}\right)\right] \\
& =\quad \sum_{k=1}^{\max _{j} l_{j}}\left[\sum_{i=1}^{n(k)-1} \phi^{C_{r}}\left(x^{\sum_{j=1}^{k-1} n(j)+i}\right)\left(Y_{\sigma_{r}\left(\sum_{j=1}^{k-1} n(j)+i\right)}-Y_{\sigma_{r}\left(\sum_{j=1}^{k-1} n(j)+i+1\right)}\right)+\right. \\
& \left.\phi^{C_{r}}\left(x^{\sum_{j=1}^{k} n(j)}\right)\left(Y_{\sigma_{r}\left(\sum_{j=1}^{k} n(j)\right)}-Y_{\sigma_{r}\left(\sum_{j=1}^{k} n(j)+1\right)}\right)\right]
\end{aligned}
$$

where $\phi^{C_{r}}$ is the payoff vector corresponding to $C_{r}$, for which $\phi^{C_{r}} \equiv v$ on $C_{r}$. But this contradicts the fact that $\phi^{C_{r}}$ is element of the Weber set. Hence, $\phi \in \mathcal{W}(v)$.

We are now in position to give the main result of the paper.

Theorem 5 For any convex multichoice game $v$, the following holds.

(i) $\mathcal{C}(v)=\mathcal{W}(v)$, or equivalently $\operatorname{Ext}(\mathcal{C}(v))=\mathcal{M}(v)$

(ii) $\mathcal{P C}^{F}(v)=\mathcal{P} \mathcal{W}(v)$, or equivalently $\operatorname{Ext}(\mathcal{P C}(v))=\mathcal{P} \mathcal{M}(v)$.

Proof: By Th. 2, we know that for a convex game $v$, any vertex of the Weber set is a vertex of the core. Since these are convex sets, and since the core is included in the Weber set by Th. 4, it follows that the vertices of the two sets coincide. For the precore, using Th. 1 and 3 similarly proves the result.

Fig. 1 summarizes most of the results on the core and the Weber set. 

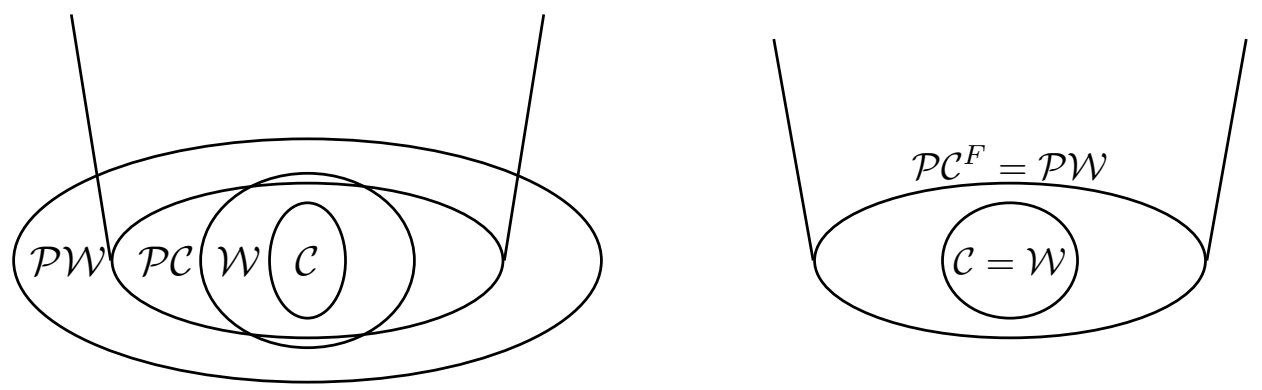

Figure 1: Relations between the core and the Weber set: general case (left), convex case (right)

\section{Comparison with previous works}

Previous works on the core of multichoice games have been done mainly by van den Nouweland et al. [14], and Faigle [5]. We summarize their approach and make a comparison with ours.

\subsection{Core of van den Nouweland}

Van den Nouweland et al. define the core of multichoice game as follows. Let $v$ be a multichoice game on $L$. A level payoff vector on $L$ is a function $\delta: M \rightarrow \mathbb{R}$, where, for all $i \in N$ and $k \in L_{i} \backslash\{0\}, \delta(i, k)$ denotes the increase in worth for player $i$ corresponding to a change of activity from level $k-1$ to level $k$ by this player, and $\delta(i, 0)=0$ for all $i \in N$.

To each $\delta$ we can associate bijectively an additive game on $L$ by

$$
\phi_{\delta}(x):=\sum_{i \in N} \sum_{k=0}^{x_{i}} \delta(i, k), \quad \forall x \in L .
$$

Due to Remark 1, $\phi_{\delta}$ can be considered as a payoff vector in our sense. Hence, in the sequel we will use indifferently the level payoff vector $\delta$ or its corresponding additive game $\phi_{\delta}$.

A level payoff vector $\delta$ on $L$ is called efficient for $v$ if $\phi_{\delta}(\top)=v(T)$, and it is called level increase rational for $v$ if, for all $i \in N$ and $k \in L_{i} \backslash\{0\}, \delta(i, k)$ is at least the increase in worth that player $i$ can obtain when he works alone and changes his activity from level $k-1$ to level $k$, i.e.

$$
\delta(i, k) \geq v\left(0_{-i}, k_{i}\right)-v\left(0_{-i},(k-1)_{i}\right)
$$

Definition 10 [14] A level payoff vector on $L$ is a level imputation of $v$ if it is efficient and level increase rational for $v$.

We denote the set of level imputations of the games $v$ by $\mathcal{I}(v)$.

Definition 11 [14] The core $\mathcal{C}_{\mathrm{VDN}}(v)$ of the game $v$ consists of all $\delta \in \mathcal{I}(v)$ that satisfy $\phi_{\delta}(x) \geq v(x)$ for all $x \in L$. 
Let $\phi_{\delta} \in \mathcal{C}_{\mathrm{VDN}}(v)$, then it satisfies $\phi_{\delta}(x) \geq v(x), \phi_{\delta}(\top)=v(\top)$. Therefore $\phi_{\delta} \in$ $\mathcal{P C}(v)$, which proves that $\mathcal{C}_{\mathrm{VDN}}(v) \subseteq \mathcal{P} \mathcal{C}(v)$.

Definition 12 [14] For a multichoice game $v$, the set $\mathcal{C}_{\min }(v)$ of minimal core elements is the set of least elements of the core:

$$
\begin{aligned}
\mathcal{C}_{\min }(v):=\left\{\phi_{\delta} \in \mathcal{C}_{\mathrm{VDN}}(v) \mid \text { there is no } \phi_{\delta^{\prime}} \in \mathcal{C}_{\mathrm{VDN}}(v) \text { such that } \delta^{\prime} \neq \delta\right. \text { and } \\
\\
\left.\phi_{\delta^{\prime}}(x) \leq \phi_{\delta}(x), \forall x \in L\right\} .
\end{aligned}
$$

Let $\mathcal{L}(v)=\left\{\phi \in \mathbb{R}^{M} \mid \phi\left(0_{-i}, k_{i}\right)-\phi\left(0_{-i},(k-1)_{i}\right) \geq v\left(0_{-i}, k_{i}\right)-v\left(0_{-i},(k-1)_{i}\right), k=\right.$ $\left.1, \ldots, l_{i}\right\}$. We have clearly: $\mathcal{P C}(v) \cap \mathcal{L}(v)=\mathcal{C}_{\mathrm{VDN}}(v)$.

In summary, we have the following inclusions.

Proposition 6 For any multichoice game $v$ on L, the following holds.

$$
\mathcal{C}(v) \cap \mathcal{L}(v) \subseteq \mathcal{C}_{\mathrm{VDN}}(v)=\mathcal{P C}(v) \cap \mathcal{L}(v) \subseteq \mathcal{P C}(v)
$$

Van den Nouweland et al. define the Weber set as follows. An admissible ordering (for $v$ ) is a bijection $\pi: M^{*} \rightarrow\left\{1, \ldots, \sum_{i \in N} l_{i}\right\}$ satisfying

$$
\pi(i, k)<\pi(i, k+1), \quad i \in N, \quad k=1, \ldots, l_{i}-1
$$

This amounts to define a maximal chain $\left\{\perp, x_{\pi}^{1}, \ldots, x_{\pi}^{\sum_{i \in N} l_{i}}\right\}$ in $L$, where $x_{\pi}^{k}$ is the element of $L$ obtained after $k$ steps according to $\pi$. The marginal vector $w^{\pi}$ associated to $\pi$ is defined as

$$
w_{(i, k)}^{\pi}=v\left(x_{\pi}^{\pi(i, k)}\right)-v\left(x_{\pi}^{\pi(i, k)-1}\right), \quad(i, k) \in M^{*} .
$$

Considering $w^{\pi}$ as a level payoff vector, we can associate the additive game $\phi_{w^{\pi}}$. The level payoff vector is a level imputation if the game is monotonic after zero-normalization (i.e., such that $v\left(0_{-i}, k_{i}\right)=0$, for all $\left.i, k\right)$. Then the Weber set in the sense of van den Nouweland is defined as the convex hull of all marginal vectors.

In fact, $\phi_{w^{\pi}}$ is exactly our additive game $\phi^{C}$, where $C$ is the maximal chain induced by $\pi$. Hence, $\mathcal{W}_{\mathrm{VDN}}(v)=\mathcal{P} \mathcal{W}(v)$.

Van den Nouweland et al. [14] showed that if $v$ is convex, then $\mathcal{W}_{\mathrm{VDN}}(v)$ is the convex hull of $\mathcal{C}_{\text {min }}$. Comparing to our results, we have proven that $\mathcal{P} \mathcal{W}(v)$ is the convex hull of the extreme points of $\mathcal{P C}(v)$. This shows that the extreme points of $\mathcal{P C}(v)$ are all minimal core elements. However, not all minimal core elements are extreme points as the following example shows.

ExAmple 6: (borrowed from [14, Ex. 3]) Let us consider a game with 2 players, $l_{1}=2, l_{2}=1$, and a convex game $v$ defined as follows:

\begin{tabular}{|c|c||c|c|}
\hline$x$ & $v(x)$ & $x$ & $v(x)$ \\
\hline$(0,0)$ & 0 & $(0,1)$ & 0 \\
$(1,0)$ & 0 & $(1,1)$ & 2 \\
$(2,0)$ & 0 & $(2,1)$ & 3 \\
\hline
\end{tabular}

There are 3 maximal chains: $C_{1}:=\{(0,0),(1,0),(2,0),(2,1)\}$, $C_{2}:=\{(0,0),(1,0),(1,1),(2,1)\}$ and $C_{3}:=\{(0,0),(0,1),(1,1),(2,1)\}$, leading to the three following additive games $\phi^{1}, \phi^{2}, \phi^{3}$ : 


\begin{tabular}{|l|l|l|}
\hline$\phi^{1}(1,0)=0$ & $\phi^{2}(1,0)=0$ & $\phi^{3}(1,0)=2$ \\
$\phi^{1}(2,0)=0$ & $\phi^{2}(2,0)=1$ & $\phi^{3}(2,0)=3$ \\
$\phi^{1}(0,1)=3$ & $\phi^{2}(0,1)=2$ & $\phi^{3}(0,1)=0$ \\
\hline
\end{tabular}

Clearly, these are all minimal core elements as expected. But the following additive game is a minimal core element:

$$
\phi^{4}(1,0)=1, \quad \phi^{4}(2,0)=2, \quad \phi^{4}(0,1)=1
$$

and is not among the three additive games of $\mathcal{P} \mathcal{M}(v)$.

This shows that $\mathcal{C}_{\text {min }}$ is unnecessarily large, since it contains elements which are not extreme points. Finally, we prove the following.

Proposition 7 For any convex game $v, \mathcal{P C}^{F}(v) \subseteq \mathcal{C}_{\mathrm{VDN}}(v)$. In other words, any convex combination of marginal worth vectors is level increase rational for a convex game $v$.

Proof: Consider $\phi^{C}$ in $\mathcal{P} \mathcal{M}(v)$, for some maximal chain $C=\left\{\perp, x^{1}, \ldots, x^{\sum_{j=1}^{n}} l_{j}=\top\right\}$. Using previous notations, the associated chain vector is $\psi^{C}$, with

$$
\psi_{i}^{C}=v\left(x^{i}\right)-v\left(x^{i-1}\right), \quad i=1, \ldots, \sum_{j=1}^{n} l_{j} .
$$

Consider $i_{j}$ in $1, \ldots, \sum_{j=1}^{n} l_{j}$ such that $x_{j}^{i_{j}}=k$ and $x_{j}^{i_{j}-1}=k-1$ for some level $k$. By convexity of $v$, we have:

$$
\psi_{i_{j}}^{C}=v\left(x^{i_{j}}\right)-v\left(x^{i_{j}-1}\right) \geq v\left(0_{-j}, k_{j}\right)-v\left(0_{-j},(k-1)_{j}\right)
$$

This being true for each $i_{j}$, we have proven that the level payoff vector $\psi^{C}$ is level increase rational. Since convex combinations preserves the property of level increase rationality, and since $\mathcal{P C}^{F}(v)$ is the convex hull of $\mathcal{P} \mathcal{M}(v)$ by Th. 5, the proof is complete.

\subsection{Core of Faigle}

U. Faigle introduced the core of games with restricted cooperation in [5]. He proposes a general model for a cooperative game on a finite set $N$ of players, without assuming that every coalition $S \subseteq N$ of players is feasible, and thus takes into account the situation, for instance, where some players may join a coalition only if some other players have already joined the coalition.

Definition 13 [5] A (finite) game with restricted cooperation is a quadruple $\gamma=\left\{N, F, v, v_{0}\right\}$ where $N$ is the finite set of players, $F$ a nonempty collection of subsets of $N$ called feasible coalitions, $v: F \rightarrow \mathbb{R}$ the value function, with $v(\emptyset)=0$, and $v_{0} \in \mathbb{R}$ the total value of the game $\gamma$. If $v$ and $v_{0}$ are nonnegative, $\gamma$ is a positive game. 
A solution of the game $\gamma$ is a fair distribution of its value $v_{0}$ among the players. As usual, they therefore define the $\operatorname{core} \mathcal{C}_{\text {Faigle }}(v)$ of the game $\gamma$ to consist of all undominated pre-imputations, i.e. vectors $\phi \in \mathbb{R}^{N}$ such that:

(i) $\sum_{s \in A} \phi_{s} \geq v(A)$ for all $A \in F$,

(ii) $\sum_{s \in N} \phi_{s}=v_{0}$.

The usual way to define the restricted set of coalitions $F$ is to introduce a partial order $\leq$ on $N$ [6]. Let $P=(N, \leq)$ denote this partially ordered set of players. The relation $i \leq j$ indicates that the presence of $j$ enforces the presence of $i$ in any coalition $S \subseteq N$. Hence, a (feasible) coalition of $P$ is a subset $S \subseteq N$ such that $s \in S$ and $t \leq s$ yield $t \in S$ for all $s, t \in N$. Thus unions and intersections of coalitions are again coalitions, while complements of coalitions may fail to have this property. By $\Gamma(P)$ we denote the vector space of all cooperative games on $P$.

Let $v$ be a multichoice game on $L$, keeping our previous notations, and consider as a set of virtual players the disjoint union $N^{\prime}=\cup_{i \in N}\left(L_{i} \backslash\left\{0_{i}\right\}\right)$, with the ordering $\leq$ defined by $n_{1} \leq n_{2}$ if $n_{1}, n_{2} \in L_{i}$ for some $i$ and $n_{1} \leq n_{2}$ in $L_{i}$. There is a one-to-one correspondence between the participation profiles $x \in L=\prod_{i=1}^{n} L_{i}$ and the feasible coalitions on $N^{\prime}$ in the sense of Faigle: the non-zero components of such an $x$ may be interpreted as the maximal elements of a feasible coalition of $N^{\prime}$ and conversely, while the zero-vector $0 \in L$ corresponds to the empty feasible coalition of $N^{\prime}$. Hence any multichoice game can be considered as a particular game with precedence constraints, on a virtual set of players $N^{\prime}$.

Let $x=\left(x_{1}, \ldots, x_{n}\right) \in L \backslash\{\perp\}$, and consider $S:=\left\{s \in N^{\prime} \mid \exists i \in N\right.$, s.t. $\left.s \leq x_{i}\right\}$. Then $S$ is a feasible coalition of $N^{\prime}$. Taking any $\phi \in \mathbb{R}^{N^{\prime}}$ belonging to $\mathcal{C}_{\text {Faigle, }}$, we have $\sum_{s \in S} \phi_{s} \geq v(x)$ and $\sum_{s \in N^{\prime}} \phi_{s}=v(\top)$, which entails that the corresponding additive game $\Phi$ belongs to $\mathcal{P C}(v)$, and reciprocally. This proves $\mathcal{P C}(v)=\mathcal{C}_{\text {Faigle }}(v)$.

\section{References}

[1] J. M. Bilbao. Cooperative games on combinatorial structures. Kluwer Academic Publishers, 2000.

[2] D. Butnariu and E. P. Klement. Triangular norm-based measures and games with fuzzy coalitions. Kluwer Academic Publishers, 1993.

[3] I. Curiel. Cooperative Game Theory and Applications. Kluwer Academic Publ., 1997.

[4] J.J.M. Derks. A short proof of the inclusion of the core in the Weber set. Int. J. of Game Theory, 21:149-150, 1992.

[5] U. Faigle. Cores with restricted cooperation. ZOR - Methods and Models of Operations Research, pages 405-422, 1989.

[6] U. Faigle and W. Kern. The Shapley value for cooperative games under precedence constraints. Int. J. of Game Theory, 21:249-266, 1992.

[7] D. Felsenthal and M. Machover. Ternary voting games. Int. J. of Game Theory, 26:335-351, 1997. 
[8] M. Grabisch. An axiomatization of the Shapley value and interaction index for games on lattices. In SCIS-ISIS 2004, 2nd Int. Conf. on Soft Computing and Intelligent Systems and 5th Int. Symp. on Advanced Intelligent Systems, Yokohama, Japan, September 2004.

[9] M. Grabisch and F. Lange. Games on lattices, multichoice games and the Shapley value: a new approach. Mathematical Methods of Operations Research, to appear.

[10] C. R. Hsiao and T. E. S. Raghavan. Multichoice cooperative games. In B. Dutta, editor, Proc. of the Int. Conf. on Game Theory and Economic Applications, New Delhi, India, 1990.

[11] C. R. Hsiao and T. E. S. Raghavan. Shapley value for multichoice cooperative games, I. Games and Economic Behavior, 5:240-256, 1993.

[12] D. Schmeidler. The nucleolus of a characteristic function game. SIAM J. on Applied Mathematics, 17:1163-1170, 1969.

[13] L. S. Shapley. A value for $n$-person games. In H. W. Kuhn and A. W. Tucker, editors, Contributions to the Theory of Games, Vol. II, number 28 in Annals of Mathematics Studies, pages 307-317. Princeton University Press, 1953.

[14] A. van den Nouweland, S. Tijs, J. Potters, and J. Zarzuelo. Cores and related solution concepts for multi-choice games. ZOR - Mathematical Methods of Operations Research, 41:289-311, 1995.

[15] L. J. Xie and M. Grabisch. The core of capacities on lattices, June 2005. Presented at the 4th Logic, Game Theory and Social Choice meeting, Caen, France.

[16] G. Ziegler. Lectures on polytopes. Springer Verlag, 1995. 